\title{
Praticando a Extensão para Promover Inclusão Digital com Computação Desplugada e Pensamento Computacional
}

\author{
Rafaela da Silva Bobsin \\ Instituto Federal do Rio Grande do \\ Sul - IFRS \\ Osório, RS, Brasil \\ rafaela.bobsin03@gmail.com
}

\author{
Natália Bernardo Nunes \\ Instituto Federal do Rio Grande do Sul - IFRS \\ Osório, RS, Brasil \\ nataliabernunes@gmail.com
}

\author{
Vithória Silveira Batista \\ Instituto Federal do Rio Grande do \\ Sul - IFRS \\ Osório, RS, Brasil \\ vithoria.sbatista@gmail.com
}

\author{
Vitória de Souza Fabrício \\ Instituto Federal do Rio Grande do \\ Sul - IFRS \\ Osório, RS, Brasil \\ vitoria.souza.fabricio@gmail.com
}

\author{
Anelise Lemke Kologeski \\ Instituto Federal do Rio Grande do Sul - IFRS \\ Osório, RS, Brasil \\ anelise.kologeski@osorio.ifrs.edu.br
}

\begin{abstract}
This work presents a practical experience that offers playful workshops with plugged and unplugged computing, including logical reasoning, computational thinking and basic programming concepts. The main objective is to attend students in the final grades of elementary school, providing digital inclusion. The activities were developed with 440 participants, from 18 different institutions, between 2017 and 2019 years. The results shown improvements in the understanding of statements related to the worked themes by up to $45 \%$. In addition, digital inclusion has been provided through information and digital communication technologies applied in education, providing a great playful moment for all people involved.
\end{abstract}

\section{KEYWORDS}

Educação, Inclusão Digital, Pensamento Computacional, Oficinas Lúdicas, Tecnologia

\section{CONTEXTUALIZAÇÃO}

O desenvolvimento tecnológico na sociedade contemporânea tem influenciado diretamente nas perspectivas de cada indivíduo sobre o presente e o futuro da população. Dentre os fatores que se relacionam com as inovações da Era Digital, evidenciam-se novas estruturas sociais, onde cada vez mais podem ser encontrados ambientes automatizados, interconectados e em constante desenvolvimento, relacionando às mais diversas atividades do cotidiano com a Tecnologia da Informação (TI) ou ao Pensamento Computacional (PC). Desta forma, o uso de recursos digitais e ferramentas tecnológicas integra a sociedade, sendo algo cada vez mais essencial às pessoas da Era Digital, deixando evidente que as competências digitais são fundamentais para o desenvolvimento no Século XXI. Isso se confirma diante do conceito de "Nativos Digitais", que se refere às pessoas que nasceram após 1980 e que possuem habilidade e facilidade para utilizar as tecnologias digitais [1]. Contudo, não podemos utilizar as novas tecnologias para perpetuar as velhas práticas, no que diz respeito à Educação, uma vez que diante de tantas transformações, a Educação também está sendo reinventada, através de um modelo educativo extremamente recente, chamado de Educação 5.0, que está sendo analisado por diversos pesquisadores, como o professor José Morgado [2]. Este modelo visa a integração para além do conhecimento e da tecnologia, isto é, a utilização do PC para promover também habilidades cognitivas, como a lógica, criatividade e o trabalho em equipe, envolvendo uma compreensão cosmopolita do mundo, citado várias vezes pelo professor Morgado [2]. De acordo com Brackmann [3] o PC é uma distinta capacidade criativa, crítica e estratégica humana de saber utilizar os fundamentos da Computação nas mais diversas áreas do conhecimento, para identificar e resolver problemas de forma colaborativa e eficiente, através de passos claros. Dessa forma, iniciativas neste contexto já são realizadas em prol da educação, contribuindo para o desenvolvimento de pensamento crítico, criatividade, capacidade de raciocínio dos estudantes e Educação 5.0 como um todo.

Com a popularização da tecnologia, iniciou-se então uma busca pela Inclusão Digital para aqueles que não têm acesso aos recursos de TI. Para que essa inclusão seja de fato percebida na sociedade, a inserção do PC se faz necessária no cotidiano educacional. Contudo, o uso da tecnologia não é observado em todos os setores da sociedade. Um estudo realizado em 2015 evidencia uma problemática no cenário educacional do Brasil: de acordo com o PISA (Programa educacional de Avaliação de Alunos), nosso país apresenta a segunda pior conectividade nas escolas, quando comparado aos 35 países avaliados [4]. Outro estudo que ressalta este problema, foi desenvolvido pela plataforma Todos Pela Educação ${ }^{1}$, indicando que a maioria das escolas públicas que possuem um laboratório de Informática apresentam problemas de baixa conexão e equipamentos ultrapassados, inviabilizando o uso da Internet e de computadores.

Buscando promover inovações na educação, várias instituições procuraram a tecnologia como meio de renovação. O Centro de

${ }^{1}$ Disponível em: https://www.todospelaeducacao.org.br/ 
Inovação para a Educação Brasileira (CIEB)², fundado em 2016, desenvolve protótipos e dissemina conhecimentos para incentivar boas práticas de uso de tecnologia na educação pública básica. Atuando no apoio de formações de políticas públicas, o CIEB prototipa ferramentas educacionais articulando a educação do ensino básico e defendendo o uso da TI como forma de realizar uma transformação na educação e nos processos de aprendizagem atuais, acreditando que "todo brasileiro tem o direito a uma educação pública de qualidade, viva e instigante que o prepare para apreender as oportunidades do seu tempo" e garante que para isso, é necessário a inclusão de tecnologia em sala de aula, gerando qualidade, equidade e contemporaneidade para a educação.

Desta forma, nosso trabalho propõe o uso de diferentes recursos de PC e TI para promover a inclusão digital na região do Litoral Norte do Estado do Rio Grande do Sul, integrando a nossa instituição com a comunidade, a fim de promover a cultura da inovação na educação pública brasileira, contextualizando os participantes com as necessidades atuais da sociedade

Contudo, além da inclusão digital que é ofertada aos jovens participantes, existe outra motivação para o projeto em questão: a baixa adesão feminina em áreas relacionadas com a tecnologia. Dessa forma, a oferta das oficinas surge como incentivo para os jovens, em especial para as meninas, a fim de que elas se envolvam com a tecnologia, uma vez que historicamente a quantidade de mulheres atuantes nesta área é menor do que a quantidade de homens, e isto não é diferente no Brasil, onde apenas $17 \%$ dos profissionais que trabalham com programação são mulheres [5].

\section{TRABALHOS RELACIONADOS}

Diante do cenário apresentado, alguns países já estão incluindo disciplinas de Robótica e Programação nas salas de aula como componentes do currículo escolar dos alunos, pois acreditam no caráter interdisciplinar que o PC apresenta. Dentre esses países, o que mais se destaca é o Reino Unido, onde em 2013 o Governo do país decidiu reformular o modelo antigo de aprendizagem, que era voltado apenas para conhecimentos básicos de Informática (como criação de planilhas e utilização de editores de texto) para um currículo voltado à área da Computação e ao desenvolvimento de Algoritmos, tornando obrigatória a Programação nas escolas [6].

Por outro lado, no Brasil, a Base Nacional Comum Curricular $(\mathrm{BNCC})^{3}$ ainda não efetivou nenhuma integração entre conceitos da Informática e as matérias básicas do currículo escolar, o que evidencia a necessidade de iniciativas externas e, por isso, diversos trabalhos apresentam propostas semelhantes ao nosso projeto, como [7] e [8], que fazem uso de recursos digitais, e [9] que usa a computação desplugada, focando na educação infantil.

Contudo, nosso projeto se diferencia por atender alunos de $9^{\circ}$ ano do ensino fundamental, permitindo ao professor participar das

\footnotetext{
${ }^{2}$ Disponível em: http://cieb.net.br/

${ }^{3}$ Disponível em: http://basenacionalcomum.mec.gov.br/
}

atividades, disponibilizando os passos das oficinas tanto com o uso de recursos digitais quanto de computação desplugada, atendendo as escolas mesmo sem a disponibilidade de computadores.

\section{METODOLOGIA}

As oficinas foram divididas em: desplugadas, envolvendo computação desplugada; digitais, com o uso de computadores e recursos digitais; e hibridas, envolvendo tarefas desplugadas e digitais. Esta diversidade de oficinas surgiu com o intuito de atender a todos os alunos, oportunizando diferentes meios de aprendizado mesmo sem o uso de laboratórios de informática ou ainda para atender os casos onde a escola não apresentava condições de deslocamento até as dependências do IFRS para o uso dos computadores. O tempo de duração das oficinas variou entre 1 hora e 30 minutos, nos anos de 2017 e 2018, e 4 horas, em 2019.

O projeto iniciou no ano de 2017 com a implantação de 2 oficinas digitais, com o auxílio das plataformas internacionais e gratuitas $\mathrm{Code}^{4}$ e $\mathrm{Scratch}^{5}$ em cada uma das oficinas.

A plataforma Code oferece diversos jogos lúdicos, que envolvem noções espaciais de direita/esquerda, comandos condicionais e laços de repetição, por exemplo. As atividades selecionadas fazem uso de temas e personagens familiares aos estudantes, como "Star Wars", "Angry Birds", "A Era do Gelo”, "Frozen" e "Minecraft", dentre outros, e são atrativas por abrangerem conteúdos pertinentes à faixa etária dos alunos, utilizando comandos bem definidos para a conclusão dos objetivos de cada fase dos jogos, onde o aluno precisa utilizar programação em blocos para concluir cada objetivo proposto, utilizando apenas os comandos disponibilizados em cada fase. Esta plataforma tem sido utilizada em todas as edições do projeto, desde 2017, por ser fácil e intuitiva, bem como por ter apresentado um ótimo retorno.

Já na plataforma Scratch, cada participante teve a oportunidade de programar e criar o seu próprio jogo. Um exemplo de atividade desenvolvida foi o jogo da corrida, em que os participantes criaram o cenário, escolheram os personagens e realizaram a programação necessária para que os personagens pudessem competir através de uma corrida controlada pelo teclado. Um passo-a-passo da execução desta atividade pode ser encontrado dentro da própria plataforma, no formato de tutorial. Contudo, nos anos de 2018 e 2019 essa plataforma não foi adotada, diante da evidente dificuldade apresentada pelos alunos para trabalharem com a construção de algoritmos em apenas um único encontro.

No ano de 2019 passaram a ser realizadas também as oficinas desplugadas, contando com atividades da plataforma Code e da plataforma Pensamento Computacional ${ }^{6}$. A primeira atividade

\footnotetext{
${ }^{4}$ Disponível em: https://code.org/

${ }^{5}$ Disponível em: https://scratch.mit.edu/

${ }^{6}$ Disponível em: http://computacional.com.br/
} 
utilizada é da plataforma Code, e é chamada de "Programação com Papel Quadriculado". Ela é composta por uma série de comandos com setas e um símbolo específico que significa "pintar um quadrado", que após uma sequência de comandos executados, forma uma figura no papel quadriculado. $\mathrm{O}$ aluno também pode realizar o processo inverso, obtendo a sequência de comandos necessária para formar uma figura já pronta. A segunda atividade utilizada foi obtida da plataforma Pensamento Computacional, e é chamada de "Estacionamento Algorítmico", que tem por objetivo mover o carro " $\mathrm{X}$ " para fora de um tabuleiro, que representa um estacionamento, devendo o aluno realizar deslocamentos específicos com os outros veículos presentes no tabuleiro que obstruem a passagem. Estes comandos devem ser anotados de forma sequencial por meio de uma tabela, e formam uma sequência de passos, como em um algoritmo.

Ainda em fase de testes, a oficina híbrida foi então implantada, para introduzir os conceitos básicos de lógica e raciocínio lógico de forma desplugada, com o uso do jogo "Desafio Genial da Turma da Mônica”, da empresa Xalingo. Ele tem como principal objetivo solucionar desafios conectando objetos e personagens específicos através de 9 peças que se encaixam, formando caminhos.

Também, durante o ano de 2019, com a realização de dois encontros de aproximadamente 4 horas cada um, observou-se que alguns alunos apresentaram muita facilidade na realização das atividades que envolviam o uso da programação em blocos, e por isso decidiu-se então aumentar a complexidade das atividades propostas, optando-se pela inserção da programação básica através da plataforma Portugol Studio ${ }^{7}$, para complementar a oficina híbrida. Ela consiste em uma ferramenta para o desenvolvimento de programação básica, através de pseudoalgoritmos desenvolvidos em português com interface didática e abordagens criadas especialmente para iniciantes. Com o Portugol Studio, os estudantes podem criar desde algoritmos simples até jogos completos, totalmente em português. A linguagem é baseada em C e PHP, e simplifica muitas funcionalidades gráficas e matemáticas, além de contar com vários exemplos. O aprendizado de pseudocódigos, em português, serve para um primeiro contato com linguagens de desenvolvimento de algoritmos, de tal forma que esse contato pode despertar no aluno algum interesse pelo tema abordado. Contudo, o objetivo desta modalidade de oficina não é capacitar um estudante a ser programador de fato, mas sim instigá-lo a ter interesse pela área tecnológica, bem como fazê-lo compreender que podem existir múltiplas soluções para um determinado problema, através de uma sequência bem definida de passos, que pode ser devidamente associada a um algoritmo.

Para o agendamento das oficinas, cada bolsista do projeto ficou responsável por procurar escolas interessadas em participar das oficinas propostas. Os encontros foram agendados conforme a disponibilidade das escolas, e foram realizados tanto nas escolas, quanto na instituição de ensino promotora, conforme o interesse e

\footnotetext{
${ }^{7}$ Disponível em: http://lite.acad.univali.br/portugol/
}

viabilidade de cada escola convidada. Desta forma, as oficinas foram organizadas em 2017 com 2 encontros de 1 hora e 30 minutos, onde num deles trabalhou-se a plataforma Code, e no outro, a plataforma Scratch. Em 2018, apenas um encontro foi realizado, de 1 hora e 30 minutos, com o uso da plataforma Code. E no ano de 2019, cada instituição participou de 2 encontros, sendo eles divididos em oficina digital, oficina desplugada ou oficina híbrida, com até 4 horas de duração. A escolha da execução de apenas 2 encontros dentre os 3 disponíveis se deu diante dos recursos disponibilizados em cada oportunidade.

Além disso, cada oficina é inicializada com a realização de um teste, chamado de "pré-teste", que tem por objetivo analisar o nível de conhecimento prévio dos alunos, antes da realização da oficina. Após o término da oficina, outro teste é realizado, chamado de "pós-teste", possibilitando analisar o desempenho obtido pelo aluno durante as atividades desenvolvidas. Cada teste é composto por 5 questões, e para as oficinas desplugadas e digitais, o mesmo teste foi aplicado no início e no final, oportunizando ao aluno corrigir as questões que havia errado inicialmente. Contudo, diante de diversas sugestões recebidas, para a oficina híbrida, optou-se por utilizar testes diferentes, também com 5 questões.

\section{RESULTADOS}

Até o presente momento foram atendidas 18 instituições distintas, contando com a presença de 440 participantes. Destes participantes 59 foram no ano de 2017, 142 em 2018 e 239 em 2019. Os resultados foram obtidos através do comparativo entre pré e pós-teste, e são apresentados na Tabela I. No ano de 2017, as oficinas digitais com a plataforma Code apresentaram uma melhoria nos resultados de apenas $2,1 \%$, enquanto que as oficinas digitais com o uso da plataforma Scratch apresentaram uma melhoria total de $11,9 \%$. Acredita-se que neste primeiro ano de execução do projeto o resultado tenha sido relativamente baixo pois foi uma primeira experiência, sendo o primeiro contato da equipe com o uso das plataformas citadas e com a preparação e condução das oficinas. Além disso, também foi observado que, normalmente, as oficinas com o uso do Scratch, em outros projetos similares ao nosso, ocorriam com múltiplos encontros, em um período de duração maior do que 1 hora e 30 minutos, e por isso a equipe de execução não utilizou esta plataforma nos anos seguintes, justamente por exigir um pouco mais de complexidade e conhecimento por parte dos alunos. Já no ano de 2018, a melhoria observada na oficina digital com a plataforma Code foi de $26,4 \%$, mostrando então um resultado bastante expressivo em comparação com o ano de 2017. No ano de 2019 o resultado mais significativo foi observado na modalidade híbrida, com aumento de $45 \%$ nos resultados, enquanto que a oficina digital com a plataforma Code e a oficina desplugada apresentaram respectivamente $30,4 \%$ e $14 \%$ de melhoria. Assim sendo, acreditamos que os baixos índices de melhoria da oficina desplugada se dão por ser uma atividade mais próxima do cotidiano estudantil dos participantes, e por isso eles se sentem 
menos motivados para a participação em tal oficina. Além disso, a quantidade de acertos no pré-teste para a oficina desplugada tem sido bem elevada (em torno de 3 a 4 questões por teste) e por isso o resultado final não tem uma variação tão significativa.

$\begin{array}{ccc}\text { Oficina (turmas/ano) } & \text { Participantes } & \text { Melhoria (\%) } \\ \text { Digital com Code (2/2017) } & 59 & 2,1 \\ \text { Digital com Scratch (2/2017) } & 59 & 11,9 \\ \text { Digital com Code (6/2018) } & 142 & 26,4 \\ \text { Desplugada (8/2019) } & 169 & 14,0 \\ \text { Digital com Code (10/2019) } & 190 & 30,4 \\ \text { Híbrida (2/2019) } & 32 & 45,0\end{array}$

Table 1. Síntese das oficinas realizadas

A quantidade de acertos por aluno em cada teste, no ano de 2019, é apresentada na Figura 1 para o Pré-Teste, e na Figura 2 para o Pós-Teste. Na Figura 1 a quantidade de acertos está entre 1 e 3 questões, sendo que o total de questões presentes no teste são 5. Na Figura 2 esse resultado fica entre 3 a 5 questões respondidas corretamente, demonstrando uma clara evolução na compreensão de enunciados e dos conceitos.

Outro fator importante que analisamos são as respostas obtidas através de comentários no Pós-Teste, para que os participantes deixem sua opinião. Até o momento, todos os comentários obtidos foram positivos, mostrando que os alunos gostaram das atividades ofertadas, e que mesmo diante das dificuldades enfrentadas por alguns participantes, eles conseguiram efetuar as atividades.

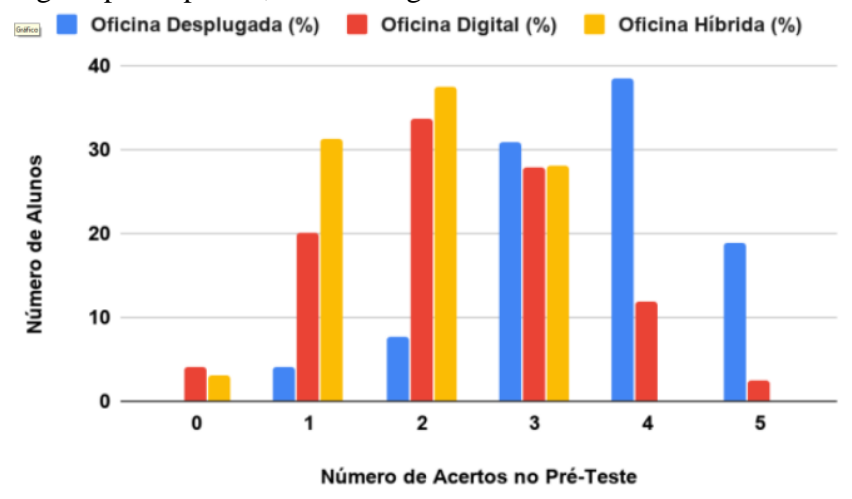

Figure 1. Número de acertos no Pré-Teste, por aluno, para cada modalidade de oficina de 2019

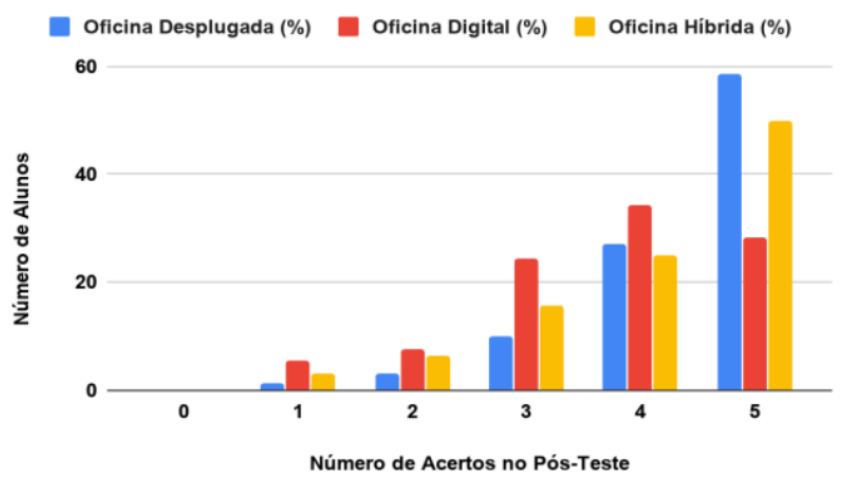

Figure 2. Número de acertos no Pós-Teste, por aluno, para cada modalidade de oficina de 2019

\section{CONCLUSÃO}

As atividades desenvolvidas pelo projeto, para o estímulo do pensamento computacional como forma de sanar as dificuldades dos alunos de escolas públicas, principalmente em relação a compreensão de texto ou em aspectos de compreensão da matemática, foram realizadas de maneira satisfatória. $\mathrm{O}$ objetivo inicial de instigar os participantes a utilizarem o raciocínio lógico para resolução de problemas do cotidiano, foi alcançado com sucesso, promovendo a inclusão digital na comunidade próxima à instituição promotora das oficinas.

Com atividades simples e lúdicas, proporcionadas por diferentes plataformas, promoveu-se a oportunidade de um aprendizado interdisciplinar ao utilizar-se da interpretação e compreensão dos enunciados propostos conjuntamente com conteúdos que envolvem o Pensamento Computacional, a Lógica de Programação, áreas da Matemática e Consciência Ambiental, por exemplo.

Para a realização das oficinas foram utilizadas atividades constituídas majoritariamente por jogos, onde os participantes precisaram seguir uma sequência de passos bem definidos para chegarem a um determinado objetivo. Através dos conteúdos abordados, os alunos observaram que a Programação e o Raciocínio Lógico estão em atividades diárias, no dia a dia de cada um.

Ao todo, foram atendidos 440 alunos, de 18 instituições, durante os anos de 2017, 2018 e 2019. O público-alvo que participou das oficinas é proveniente de turmas de $8^{\circ}$ e $9^{\circ}$ anos do ensino fundamental, com idade majoritariamente entre 14 e 16 anos. Os resultados obtidos variaram de $2,1 \%$, na primeira edição do projeto em 2017, a $45 \%$ no ano de 2019 , demonstrando uma evolução na capacidade de compreensão de enunciados pelos participantes.

Como trabalhos futuros pretende-se dar continuidade a este projeto, diversificando e incluindo mais atividades, bem como incentivando o interesse dos estudantes pela tecnologia.

\section{REFERÊNCIAS}




\section{Computer on the Beach}

[1] PALFREY, John; GASSEE, Urs. Nascidos na era digital: entendendo a primeira geração dos nativos digitais. Porto Alegre: Artmed, 2011

[2] MORGADO, José Carlos. O professor como decisor curricular: de ortodoxo a cosmopolita. Revista Tempos e Espaços em Educação, São Cristóvão, Sergipe, Brasil, v. 9, n. 18, p. 55-64, jan./abr. 2016. ISSN: 2358-1425.

[3] BRACKMANN, Christian. Desenvolvimento do Pensamento Computacional Através de Atividades Desplugadas na Educação Básica. 2017. Universidade Federal do Rio Grande do Sul (UFRGS), Porto Alegre, RS, Brasil, 2017.

[4] Folha de Londrina, 2018. Uso de Tecnologias nas escolas ainda é precário. Disponível em: <https://folhadelondrina.com.br/cadernosespeciais/uso-de-tecnologias-nas-escolas-ainda-e-precario-1001410.html $>$. Acesso em 27 de outubro de 2019.

[5] Época, 2018. Negócios. Apenas $17 \%$ dos programadores brasileiros são mulheres. $2018 . \quad$ Disponível em: $<$ https://epocanegocios.globo.com/Economia/noticia/2018/02/apenas-17-dosprogramadores-brasileiros-sao- mulheres.html> Acesso em agosto de 2019

[6] Gazeta, 2016. Naiady Piva. "Inclusão da programação nos currículos escolares avança no exterior". 2016. Disponível em: $<$ https://www.gazetadopovo.com.br/educacao/inclusao-da-programacao-noscurriculos-escolares-avanca-no-exterior-9dlmbpvkpztvrp4vp164718fk/>. Acesso em agosto de 2019

[7] NETO, Aldenor de S. L.. O uso da Linguagem de Programação Voltada para as Crianças do $9^{\circ}$ ano do Ensino Fundamental do Colégio Tiradentes da Escola de Polícia. X Simpósio Linguagens e Identidades da/na Amazônia Sul-Ocidental, 2016.

[8] GARLET, Daniela; BIGOLIN, Nara M.; SIDNEI, Renato S.. Uma Proposta para o Ensino de Programação de Computadores na Educação Básica. Universidade Federal de Santa Maria (UFSM), Santa Maria, RS, Brasil, 2016

[9] SANTOS, Elisângela R. dos; SOARES, Graciele; BIANCO; Guilherme D.; FILHO, João B. da R.; LAHM, Regis A.. Estímulo do Pensamento Computacional a partir da Computação Desplugada: uma proposta para a Educação Infantil. RELATEC - Revista Latinoamericana de Tecnología Educativa, v. 15, 2016 